\title{
Efficiency evaluation of cornea crosslinking in septic ulcers in small pets
}

\author{
U.E. Lukashina ${ }^{1, *}$, N.A. Slesarenko ${ }^{1}$, and A.G. Shilkin ${ }^{2}$ \\ ${ }^{1}$ FSBEI of HE MSAVMB named after K.I. Skryabin, Akademika Skryabina st, bld. 23, 109472 \\ Moscow, Russia \\ ${ }^{2}$ Center of Veterinary Ophthalmology of Dr. Shilkin A.G., Snezhnaya st., 13, 129323, Moscow, \\ Russia
}

\begin{abstract}
The article presents the morphological substantiation of the feasibility of using corneal collagen crosslinking, as well as comparative characteristics of standard therapeutic animal treatment with septic cornea ulcers and modified crosslinking. Methods of ophthalmological examination of small pets after modified crosslinking revealed the most favorable (in $91.2 \%$ of cases) recovery in comparison with the standard therapeutic technique, which is confirmed by keratomalacia inhibition, acceleration of corneal epithelization rate and reduction (by $40 \%$ ) of rehabilitation period of the animal.
\end{abstract}

\section{Introduction}

Pathologies of the cornea are the most common damage to the visual organ $[3,5,9]$, so there is an urgent need to introduce modern treatment methods into routine veterinary ophthalmological practice $[3,4,9,10]$. However, such severe keratopathies as deep septic ulcers [5], descemetocele complicated by keratomalacia [4] are urgent conditions that can lead to loss of visual functions of the eye or the eyeball itself in the absence of therapeutic correction.

Keratomalacia in septic corneal ulcers is dangerous by the rapid course and loss of the corneal stroma collagen matrix with its thinning [6,7]. In this regard, the speed of therapeutic intervention as well as the rapid effect of its use is important to control the course of the pathological process. However, the keratomalacia process manifestation often outstrips the achievement of the necessary drug treatment action, which can aggravate the pathological process to descemetocele or perforation with a threat of intraocular structures infection [10].

To control the course of the septic process, we use the technique of corneal collagen crosslinking, which creates additional interfibrillary bonds, making the cornea more resistant to effects of bacterial and matrix enzymes. Crosslinking also improves the biomechanical functions of the cornea $[1,8]$. In turn, this leads to corneal permeability weakening for all medicines applied after the crosslinking procedure [2].

This message summarizes the application results of the modified crosslinking method, which consists of the combined action of forced antibiotic therapy and crosslinking [2] in

\footnotetext{
*Corresponding author: 1-ylia@mail.ru
} 
comparison with standard therapeutic protocol for small pets' treatment with septic corneal ulcers [9].

The purpose of the study was to assess the effectiveness of corneal crosslinking technique application in small pets' treatment with septic corneal ulcers.

Research objectives:

1. To establish morphofunctional changes of the cornea after cross-linking

2. To assess the effectiveness of corneal collagen crosslinking use in clinical practice for the treatment of small pets with septic ulcers

3. To present a comparative effectiveness analysis of standard therapeutic treatment and modified methodology

\section{Materials and methods of research}

The work is a fragment of complex studies of the Department of Anatomy and Histology of Animals named after Professor A.F. Klimov conducted based on the Center of Veterinary Ophthalmology of Dr. Shilkin A.G. Morphological studies were performed in the Department of Pathomorphology of the FSAI NMRC "ISTC "Mikrokhirurgiya glaza" named after academician S.N. Fedorov" in the period of 2019-2020.

Corneoscleral discs of a pig's eye fixed in a $10 \%$ solution of neutral formalin were selected for morphological assessment of the crosslinking effect on the cornea. Discs were cut into two halves, the material was washed with running water, dehydrated in alcohols of rising concentration and poured into paraffin. Next, serial histological cuts were performed, which were stained with hematoxylin and eosin and studied by light microscopy using a microscope by Leica DMLV2 company with a digital camera Leica DFC320 (Germany) at x40, x200, x400 magnification and microphotography.

Small pets (cats and dogs) with severe septic corneal ulcers were chosen as targets of the clinical trial. The technique of ophthalmological examination was used for animal groups' selection, including biomicroscopy, tonometry, ophthalmoscopy, vital tests, photographic documentation.

Two groups were formed to conduct the study:

1 group - control, $(n=30)$ included 15 cats and 15 dogs given standard therapy, including local and systemic antibiotic therapy and lubricants' application (Figure 1). The average age amounted to 8.4 years (range from 5.6 years to 12 years) among dogs and 7.1 years (range from 4.5 years up to 10 years) among cats. Instillation of broad-spectrum drugs was used as local antibiotic therapy, such as fluoroquinolones - ofloxacin $3 \mathrm{mg} / \mathrm{ml}$ (eye drops "Floxal", ALCON) or moxifloxacin $0.5 \%$ (eye drops "Vigamox", ALCON), aminoglycosides tobramycin 0,3\% (eye drops "Tobrex", ALCON) once every 3-4 hours. Systemic antibiotic therapy included prescription for doxycycline at the rate of $10 \mathrm{mg} / \mathrm{kg}$ once a day for cats and dogs. In animals with reactive iridocyclitis, instillations of a cycloplegic solution were locally applied - phenylephrine 5\% and tropicamide 0,8\% (eye drops "Midrimax", SENTIS). 

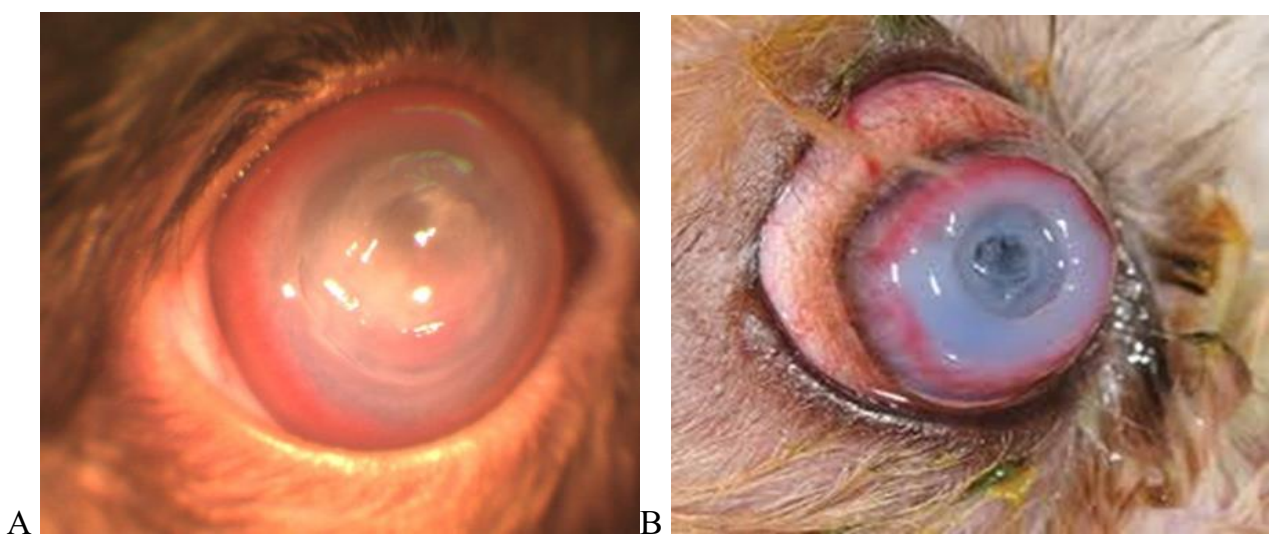

Fig. 1. A - appearance of a dog's eyeball with a deep septic corneal ulcer, B - keratomalacia complicated by descemetocele and total corneal edema.

2 group - experimental $(n=34)$ included 18 cats and 16 dogs, on which the original modified crosslinking technique was applied based on the synergistic effect of the PACKCXL protocol and forced antibiotic therapy. The average age was 7.6 years (range from 4.2 years to 13 years) among dogs and 7.5 years (range from 5 years to 11.2 years) among cats.

The automated device "UFAlink" was used for irradiation with disturbance diameters over $10 \mathrm{~mm}$ and "Apparatus for cornea phototherapy" for keratomalacia of limited (up to 10 $\mathrm{mm}$ ) diameter (Figure 2).
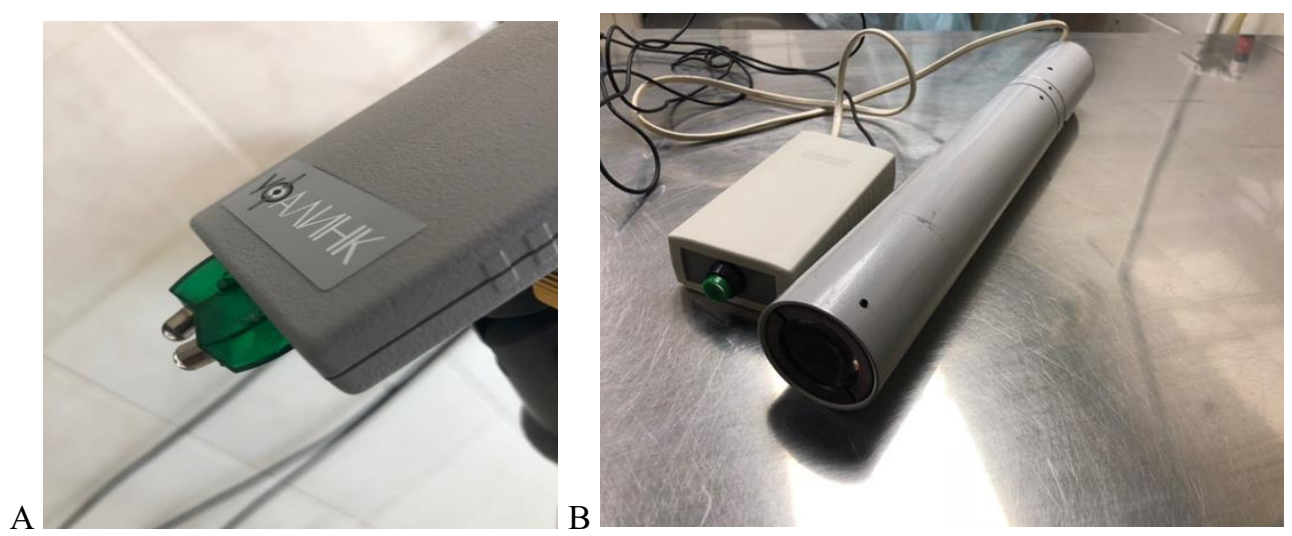

Fig. 2. Appearance of devices for corneal collagen cross-linking: A - UFAlink, B - apparatus for cornea phototherapy

To achieve the synergistic effect of antibiotic therapy and cross-linking, alternating instillation of $0.1 \%$ riboflavin solution once every 2 minutes and an antibiotic once every 3 5 minutes (Figure 3) were applied. Instillations were carried out for an hour before cornea saturation with riboflavin. Further irradiation was carried out according to the PACK-CXL protocol with a wavelength of $370 \mathrm{~nm}$, power from $5 \mathrm{MW} \backslash \mathrm{cm} 2$ to $18 \mathrm{~mW} / \mathrm{cm} 2$, exposure time - from 18 minutes to 5 minutes, respectively. During irradiation, additional instillation of riboflavin solution was performed to achieve maximum disinfectant effect by riboflavin photo activation. 


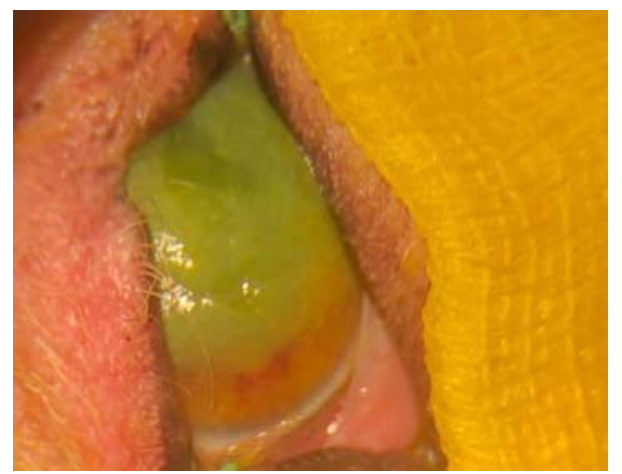

Fig. 3. Appearance of a dog's eyeball with septic corneal ulcer complicated by anterior uveitis during instillation of riboflavin solution.

The average observation period amounted to about 3 months for the control and experimental group (between 0.1-6 months). The survey during the observation period included slotted biomicroscopy, fluorescein vital test, tonometry, ophthalmoscopy and photographic documentation analysis. When the cornea melting progression and its thinning was detected in the control group, crosslinking was carried out to control keratomalacia, and with the threat of perforation - surgical stabilization by penetrating keratoplasty.

\section{Results}

Based on the study of cornea morphological features, it was found that after cross-linking it differed from the cornea that was not exposed to radiation. Thus, in the anterior stroma layers showed an increase in the density of collagen fibers' compactization without signs of edema, as well as the presence of a demarcation line separating the exposure area from the indifferent zone, which lied at various depths (from $1 / 4$ to $1 / 2$ of cornea thickness). Dense fibers packing was accompanied by a violation of layers' stratification (Figure 4 ).
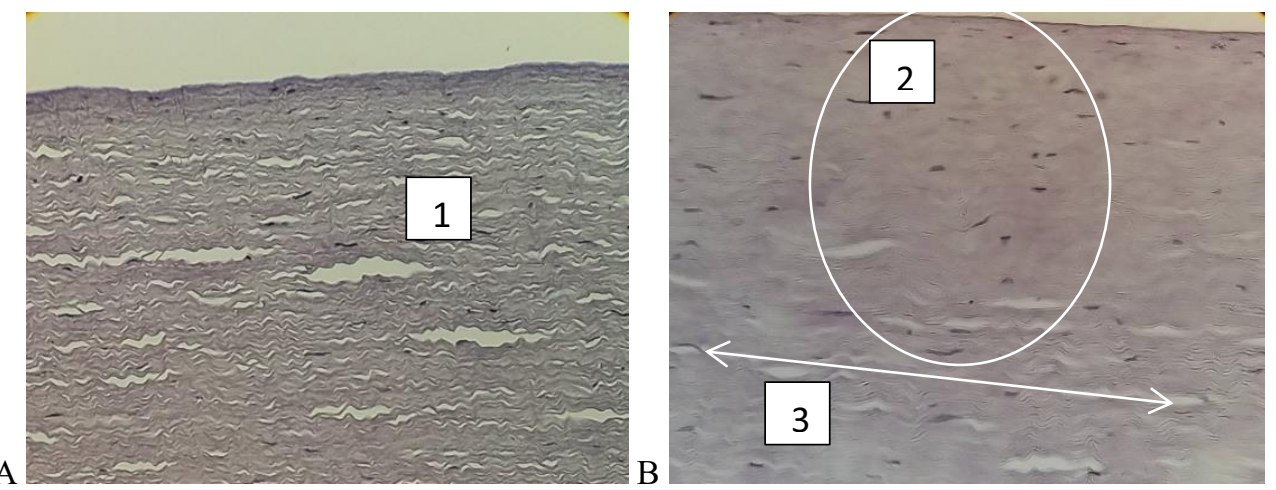

Fig. 4. Micromorphology of corneoscleral disc: A - normal cornea structure, B - cornea after crosslinking; 1 - slotted inter-fiber spaces - sign of edema, 2 - violation of layers' stratification, 3 demarcation line.

A - Hematoxylin and eosin, vol.20, c. 10

B - Hematoxylin and eosin, vol.40, c. 10 


\section{Clinical trial results}

When conducting a clinical trial in the control group (15 cats and 15 dogs), standard therapy was performed to control the course of the septic process.

A favorable outcome without additional surgical interventions with restoration of corneal stroma and complete epithelization of the defect (Figure 5) was achieved in 21 animals $(70 \%)$. The period from the treatment start to the moment of complete defect epithelization and therapy withdrawal was from 25 to 58 days (an average of 45 days).

The outcome in all these animals was corneal fibrosis of varying density degrees (from invisible to the naked eye up to opaque). In 10 dogs of brachycephalic breeds (4 pugs, 3 Pekineses, 2 Shih Tzu, 1 griffon), pigmentation of the damage zone was detected.
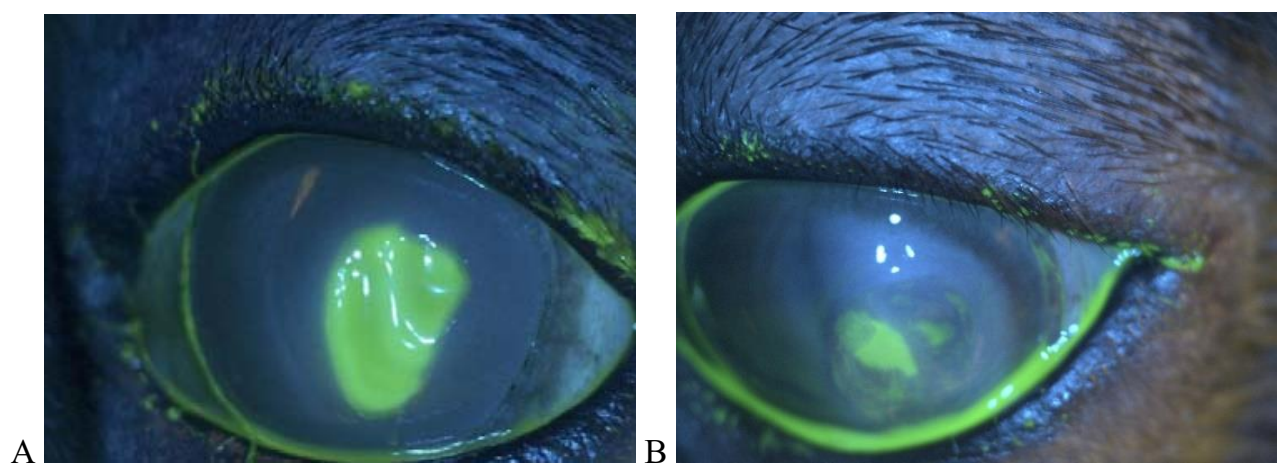

A

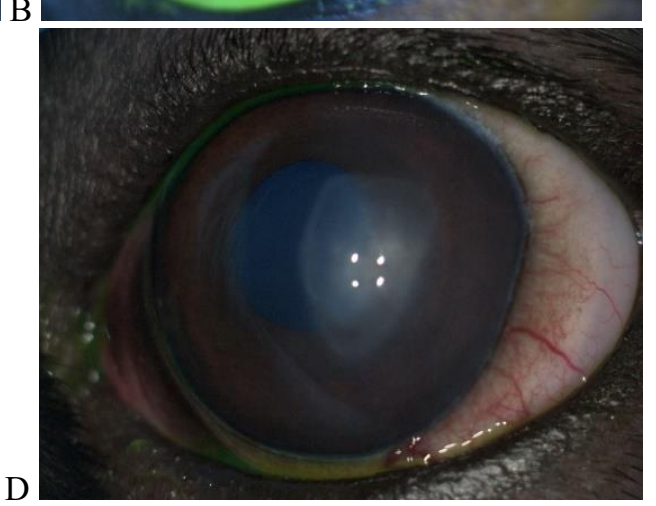

$\mathrm{C}$

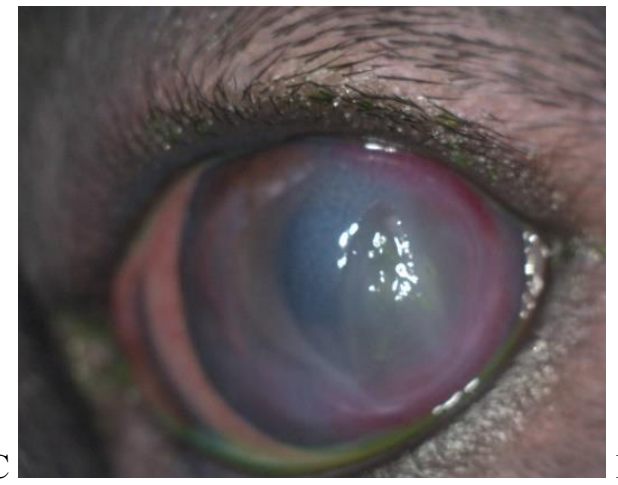

Fig. 5. Appearance of the dog's eyeball. Macromorphology of anterior corneal stroma condition during septic ulcer regeneration: A - positive vital test with fluorescein, septic corneal ulcer complicated by iridocyclitis, subtotal corneal edema, B - epithelization start, significant reduction in the defect area, vascularization of the lateral segment on the 7 th day from the therapeutic correction start C - complete epithelization, severe stromal defect, vascularization of lateral and ventral segments, preservation of corneal edema on the 14th day from the therapeutic treatment start

D - semi-translucent corneal fibrosis with single vessels on the 49th day of treatment

Complications occurred in 9 animals (30\%). All cases were represented by the progression of the pathological process. 7 animals managed to stop the septic process by carrying out modified cross-linking. In 2 animals with extremely thinned cornea, penetrating keratoplasty was carried out to stabilize it (Figure 6). The observation period from the treatment start to the detection of negative dynamics varied from 2 to 8 days. 
A
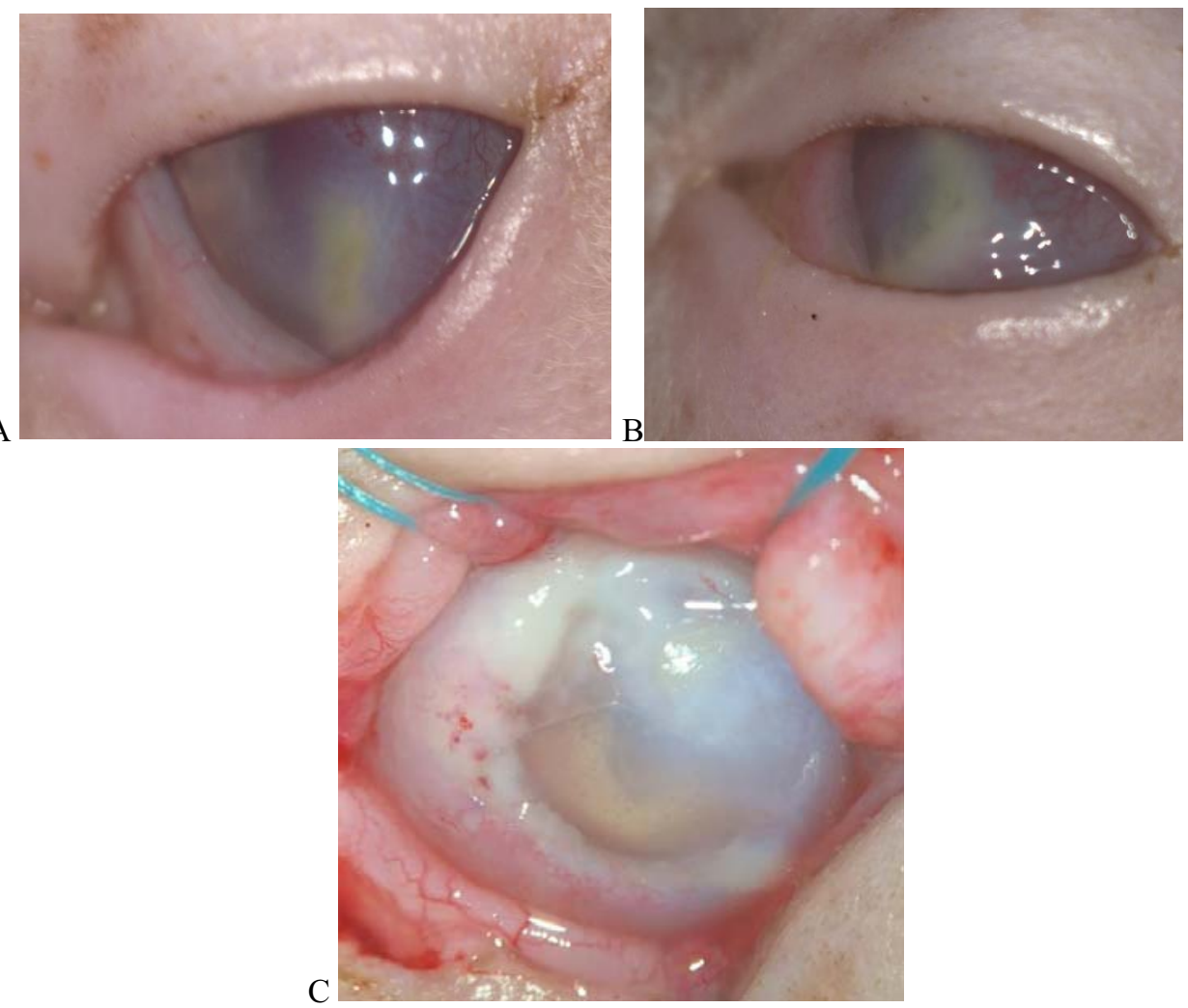

Fig. 6. Appearance of cat's eyeball with septic corneal lesion: A - purulent infiltration of the central cornea area, cornea vascularization, iridocyclite, B - increase in corneal infiltration area, total corneal edema - condition on the 2nd day of treatment, B - descemetocele with severe corneal keratomalacia, condition on the 3rd treatment day - before urgent through keratoplasty.

During a clinical trial in the experimental group (18 cats and 16 dogs), a modified crosslinking technique was performed to control the septic process.

A favorable outcome with a fully restored defect was achieved in 31 cases $(91.2 \%)$. The cornea has been completely epithelized with varying fibrosis degrees (Figure 7). Fibrosis invisible to the naked eye was achieved in 3 cats. All others had pronounced (up to opaque) fibrosis, sometimes with residual vascularization. The period from the treatment start to the moment of complete defect epithelization and therapy withdrawal was from 14 to 48 days (27 days on average). In 12 dogs of brachycephalic breeds ( 3 French bulldogs, 3 pekingeses, 3 pugs, 2 English bulldogs, 1 boxer), pigmentation of the scarring zone was detected.

A
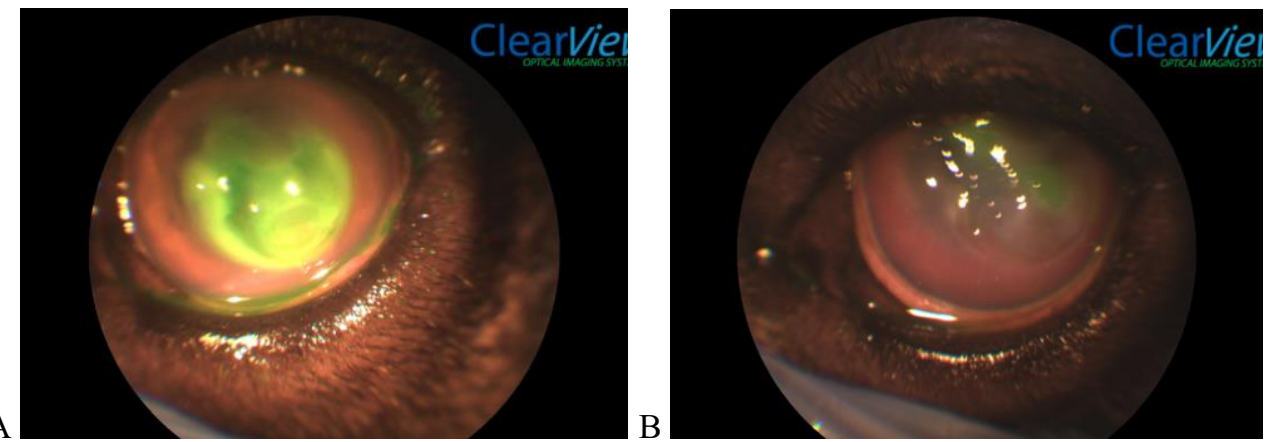

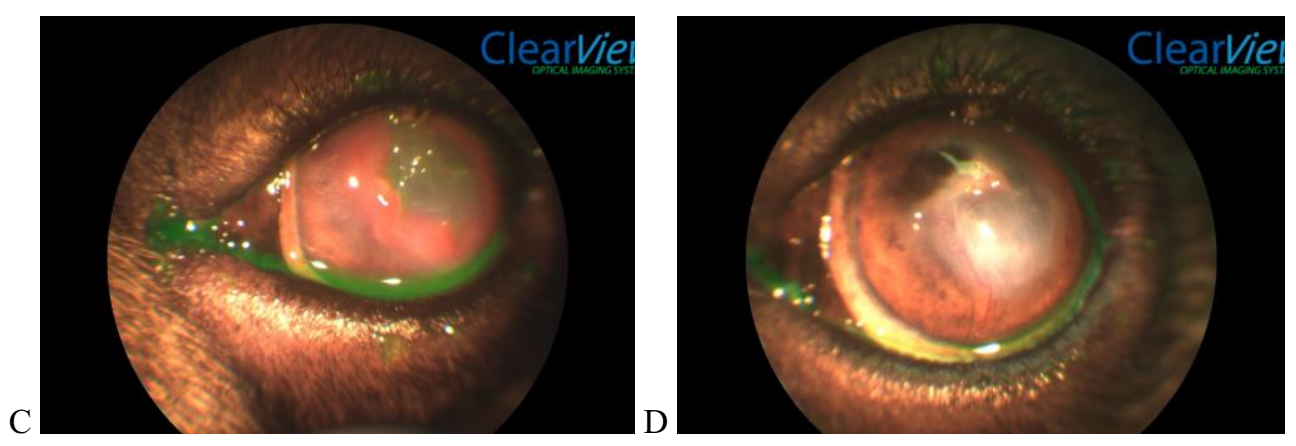

Fig. 7. A - septic corneal ulcer in a dog complicated by anterior uveitis and upper eyelid neoplasm, B - epithelization start and extensive vascularization on the 9th day after the modified cross-linking, C reduction of the stromal defect area, complete epithelization, pronounced granulation on the 21st day, D - fibrosis, residual vascularization, and cornea pigmentation on the 48th day after modified crosslinking. Photos were made using ClearView device with front segment head.

Complications occurred in 3 cases $(8.8 \%)$. The observation period before detection of cornea melting progression and thinning was from 3 to 10 days. Of these, two cases $(5.9 \%)$ required surgical stabilization to maintain tightness. In one case $(2.9 \%)$, the eye was removed due to the development of purulent endophthalmitis (Figure 8).
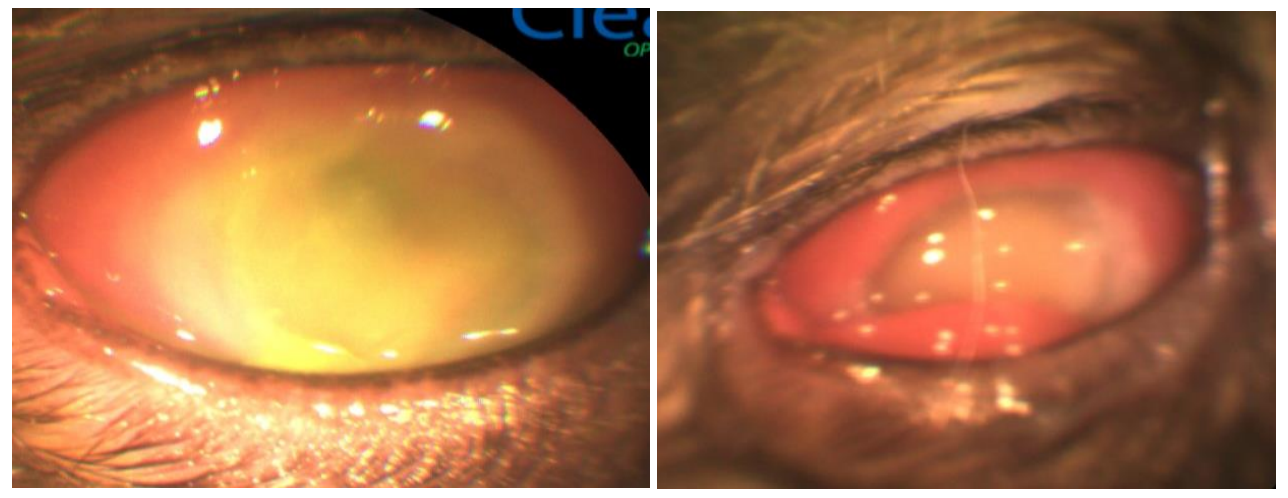

Fig. 8. Appearance of a cat's eyeball with an extensive septic corneal ulcer complicated by keratomalacia and hypopion in the anterior chamber of the eye:

A - the condition of the cornea before carrying out modified cross-linking

$\mathrm{B}$ - the eyeball condition on the 3rd day after the procedure - cornea thinning, increased vascularization, conjunctival chemosis, ocular hypertension, hypoechogenic content in the vitreous body (ultrasound diagnostics). Photos were made using ClearView device with front segment head.

\section{Deductions}

1. Morphofunctional changes in the cornea after cross-linking were revealed consisting in an increase of compositional collagen fibers density as a result of photochemical effects due to their consolidation with clear demarcation line formation limiting the impact area from the indifferent zone.

2. High (91.2\% of cases) efficiency of modified cross-linking technique application with eye tightness preservation without surgical stabilization and visual functions with fibrosis formation of varying transparency degrees were established.

3. In comparison with the standard protocol for therapeutic treatment of septic corneal ulcers, the method of modified cross-linking minimizes (by 21.2\%) the risk of complications, and 
also allows to reduce (by $40 \%$ ) the time of corneal regeneration and the period of animal treatment.

\section{Conclusion}

Comparative treatment analysis of small pets with septic corneal ulcers showed higher (21.2\%) effectiveness of modified crosslinking compared to standard method of therapeutic correction.

At the same time, after carrying out modified crosslinking, it was possible to reduce the treatment period by $40 \%$ and minimize the risk of complications (Figure 9 ).

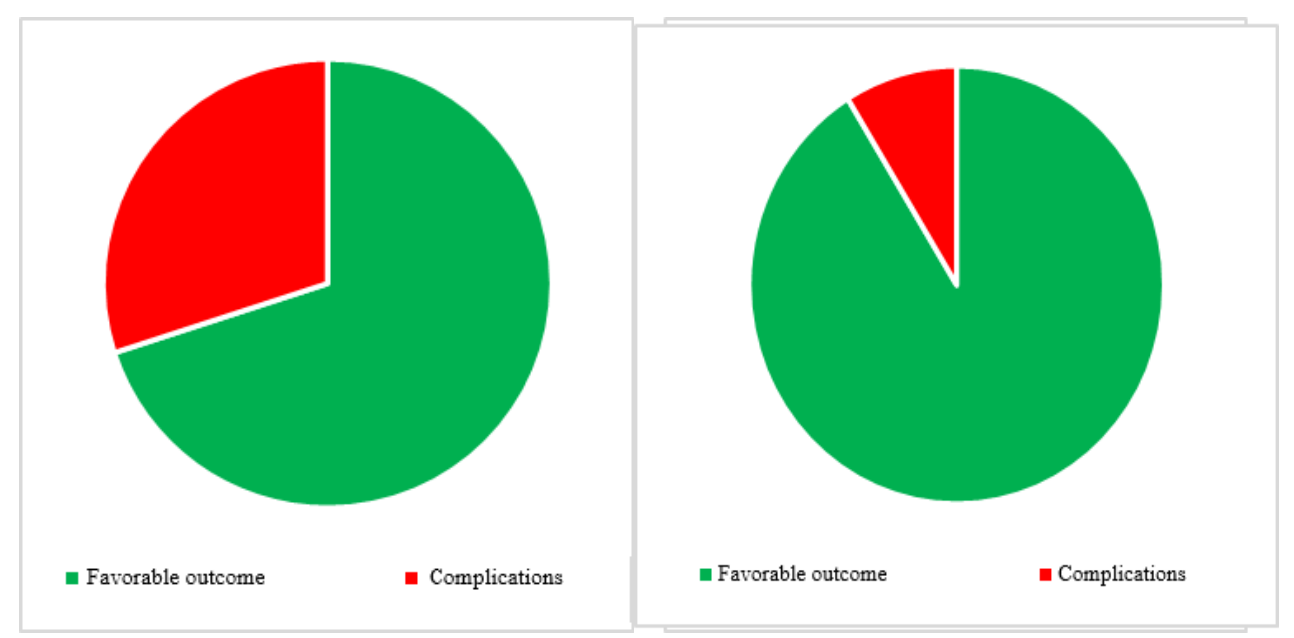

Fig. 9. Comparative analysis of the number of complications developed during the standard therapeutic treatment (A) and during the modified crosslinking method (B).

\section{References}

1. M.M. Bikbov, A.R. Khalimov, E.L. Usubov, Actual issues of ophthalmology, 71 (3), 224-232 (2016)

2. E.A. Kasparova, Ya. Bao, O.I. Sobkova, Ophthalmology, 14 (3), 274-277 (2017)

3. T.N. Pavlova, Keratoplasty in Veterinary Ophthalmology: Dissertation, 155 (2013)

4. T.N. Pavlova, Theses of the II All-Russian Inter-university Conference on Veterinary Surgery, 125-129 (2011)

5. A.G. Shilkin, Veterinary, 5, 53-55 (2004)

6. F. Famos, Veterinary Ophthalmology, 5(17), 358-367 (2014)

7. F. Famose, Veterinary Ophthalmology, 18(2), 95-104 (2015)

8. D. Said, M. Elalfy, Z. Gatzioufas, Ophthalmology, 121(7), 1377-1382 (2014)

9. M.B. Spiess, A.S. Pot, M. Florin, F. Hafezi, Veterinary Ophthalmology, 1(17), 1-11 (2014)

10. A. Pot, Veterinary ophthalmology, 4(17), 250-260 (2014) 